\title{
Ns2Voip++, an enhanced module for VolP simulations
}

\author{
(Poster Abstract) \\ Matteo Maria Andreozzi, Daniele Migliorini, Giovanni Stea, Carlo Vallati \\ Dipartimento di Ingegneria dell'Informazione \\ University of Pisa, Via Diotisalvi, 2 - 56122, Pisa, ITALY \\ \{m.andreozzi, d.migliorini, g.stea, c.vallati\}@iet.unipi.it
}

\begin{abstract}
In the last ten years, many circuit-switched networks for voice have been replaced with packet-switched ones. Hence, simulating Voice over IP has become of paramount importance in assessing the performance of a network. However, a sound performance analysis should be carried out in conditions which are as close as possible to a real deployment. In this paper we present enhancements to ns2voip [1], a module for simulating realistic VoIP traffic with the ns2 simulator. In detail, we add new features, i.e. a correlated model for packet generation in a two-way conversation and implementing a set of realistic playout buffers to simulate the behavior of the receiver. Our code is available at http://cng1.iet.unipi.it/wiki/index.php/Ns2voip.
\end{abstract}

\section{Categories and Subject Descriptors}

G.3 [Mathematics of Computing]: Probability and Statistics statistical software. I.6.7 [Computing Methodologies]: Simulation Support Systems - environments.

\section{General Terms}

Measurement, Performance, Experimentation, Verification.

\section{Keywords}

Simulation, ns-2, VoIP, MOS, QoS.

\section{INTRODUCTION}

It is well known that the performance of VoIP heavily depends on the QoS offered by the network. As such, simulations are widely used to asses its performance to ensure in a pre-deployment stage. For this reason, offsetting up meaningful and lifelike simulation scenarios is of paramount importance to get results which the deployment of new networks can rely upon. We present enhancements to the popular ns2voip [1], a module for VoIP traffic generation. This module is developed for the ns2 simulator [2] one of the most popular simulation tools for the networking community. In its first release ns2voip allows to simulate a voice application supporting: different codecs, Voice Activity Detection (VAD), and aggregation of multiple voice frames into the same IP packet.

Permission to make digital or hard copies of all or part of this work for personal or classroom use is granted without fee provided that copies are not made or distributed for profit or commercial advantage and that copies bear this notice and the full citation on the first page. To copy otherwise, or republish, to post on servers or to redistribute to lists, requires prior specific permission and/or a fee.

SIMUTools 2010 March 15-19, Torremolinos, Malaga, Spain.

Copyright 2010 ICST, ISBN 78-963-9799-87-5.
Online collections of the data that are used to evaluate the performance is done through ns2measure [6]. Among them, one of the most significant is the Mean Opinion Score (MOS) [3] which quantifies the quality of the conversation perceived by the end user. The two main limitations of this first release are i) the traffic generation model and ii) the playout buffers. Traffic generation at the source was driven only by the codec model and the state of $\mathrm{VAD}$ at the source (on or off), without considering the status of the receiver. Uncorrelated packets generation between the two sides does not consider the interplay between silence and activity periods of the two applications: most of the times the two parties will take turns in talking and listening, with relatively short transitions periods of cross-talking or mutual silence The traffic generated overlooking this interplay can be considerably different from a real conversation. The second enhancement is the implementation of playout buffers. In the first release, only an optimal, non causal playout buffer was implemented, which cannot be implemented in any real system. As this is a critical component for VoIP performance [1] evaluation would always yield overrated results. In our revised version, several real playout buffers were added, e.g. h323 [7], which is used in several SIP clients (e.g., Ekiga [4]). Hereafter we briefly describe the module focusing on the enhancements introduced in this new major revision, and we illustrate some simulation results. The latter show how more reliable results can be harvested with the new version of the module.

\section{DESCRIPTION OF THE MODULE}

The structure of the VoIP module is illustrated in Figure 1.

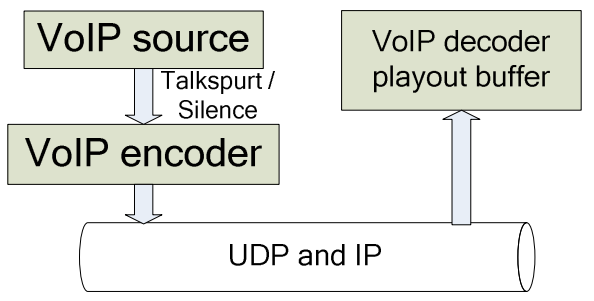

Figure 1. VoIP module structure

A VoIP source models the user activity, deciding whether a user is in a talkspurt or in a silence period. In the former case, the source signals to the encoder the beginning and the (randomly selected) talkspurt duration. During a talkspurt, the encoder generates data according to the chosen codec. The latter defines the size of each packet and the frequency of their generation. Many popular VoIP codecs are implemented (e.g., G.711 and GSM.AMR), and more can be added at negligible implementation cost. At the receiver side, a VoIP decoder receives packets from the network and employs a playout buffer to pace their playout. the MOS, as well as 
other performance metrics, are evaluated at the decoder. In the following, we describe in details the two enhancements of this new version.

\subsection{VoIP correlated traffic}

In order to model Voice Activity Detection (VAD) we implement the model described in [5]. In the latter, the whole conversation is simulated through an eight-state Markov chain, whose states represent either mutual silences (both speakers silent), or active talking periods (either or both parties talking at the same time).

\subsection{Playout buffers}

The original ns2voip release included only the optimal non-causal playout buffer. In this new release we add realistic well-known playout buffers, i.e. h323 [7] and eEM (causal) [1]. The former is a dynamic playout buffer used in many applications, e.g. Ekiga. "dynamic" means that it adapts the playout point on a pertalkspurt basis. The real H323 codec was taken from the Ekiga application source code and embedded into ns 2 voip, tweaking it to have it handle ns2voip talk frames and collect ns2measure statistics.

The eEM playout buffer can be seen as the causal reduction of the optmal one. It computes the optimal playout delay for a talkspurt (i.e., the one warranting the best achievable MOS) a posteriori. The latter is used as the playout delay for the next talkspurt, assuming that variations in the optimal playout delays of two consecutive talkspurts should be small enough.

\section{SIMULATION RESULTS}

We demonstrate how playout buffers and voice activity models influence the perceived performance. We simulated a wireless network with an access point, scheduling downlink traffic according to the Earliest Due Date (EDD) policy. Figure 2 illustrates the average MOS versus the mobile station ID. As expected, the optimal playout buffer offers the highest performance, and its causal reduction provides the worst. The results show that the difference between these two solutions is of $20 \%$ on average, which is a relevant figure. Figure 3 shows the differences in the MOS for different sets of users, obtained using uncorrelated and correlated (bi-directional) VoIP streams. In the latter case the model takes into account mutual silence states and reduces cross-talk states. Talk activity of correlated flows is generally lower than uncorrelated ones, which leads to different and more accurate simulation results. For instance, saturation occurs considerably later when using the bi-directional model.

\section{CONCLUSIONS}

In this work we present a new revision for the ns2VoIP software package. A new set of playout buffers and a correlated traffic source model has been implemented in order to enhance the simulations reliability.

\section{ACKNOWLEDGMENTS}

The authors would like to thank Luca Niccolini for his valuable help in implementing the new VoIP source model.

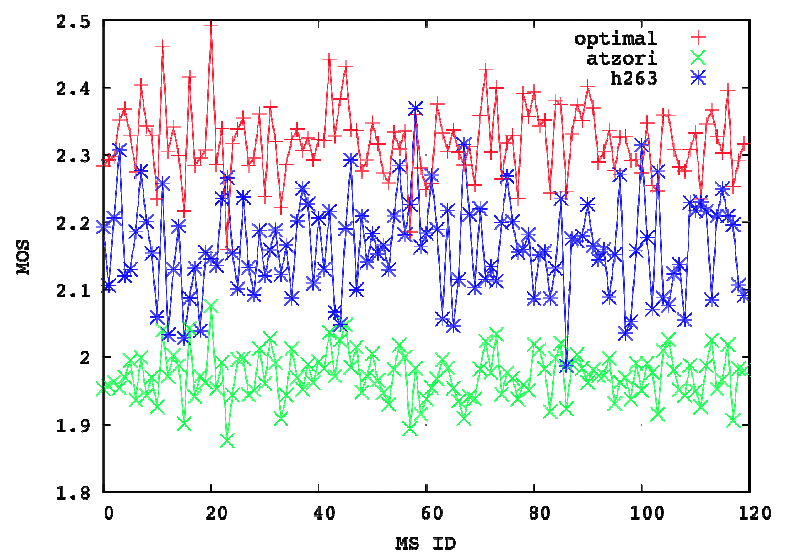

Figure 2. MOS with different PO buffers

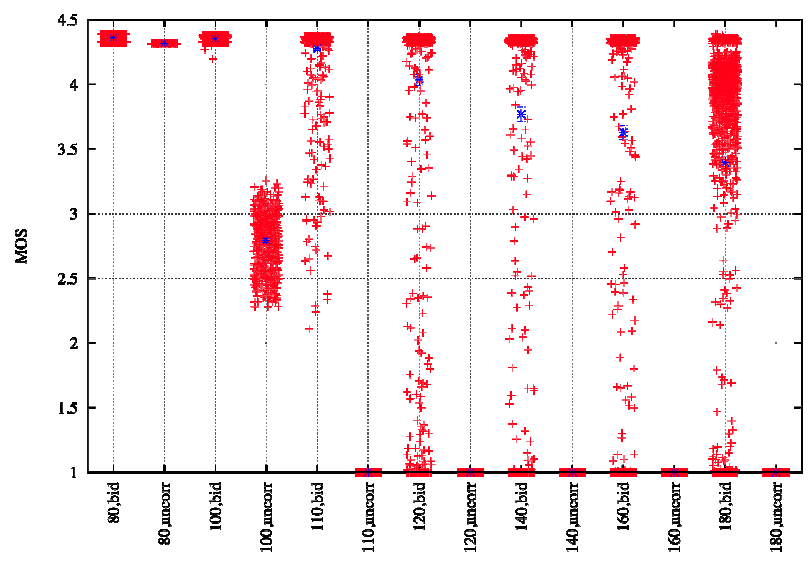

Figure 3. MOS distribution near the saturation point

\section{REFERENCES}

[1] Bacioccola, A., Cicconetti, C., and Stea, G. 2007. User-level performance evaluation of VoIP using ns-2. In Proceedings of the 2nd international Conference on Performance Evaluation Methodologies and Tools. ValueTools. ICST.

[2] N2 project home page, http://www.isi.edu/nsnam/ns/.

[3] Cole, R. G. and Rosenbluth, J. H. "Voice over IP performance monitoring". SIGCOMM Computer Communication Review, 2001

[4] Ekiga, SIP client, http://www.ekiga.org/.

[5] Stern, H.P., Mahmoud, S.A., Wong, K., "A Comprehensive Model for Voice Activity in Conversational SpeechDevelopment and Application to Performance Analysis of New-Generation Wireless Communication Systems," Wireless Networks (Springer).

[6] Cicconetti, C., Mingozzi, E., and Stea, G., “An integrated framework for enabling effective data collection and statistical analysis with ns-2". In Proceeding of WNS2'06 (Pisa, Italy, October 10, 2006).

[7] http://www.h323plus.org/, accessed Dec. 2009

[8] Atzori, L., Lobina, M.L., Corona, M., "Playout buffering of speech packets based on a quality maximization approach," IEEE Transactions on Multimedia . 